\title{
THE SUBCLASS ALGEBRA ASSOCIATED WITH A FINITE GROUP AND SUBGROUP
}

\author{
BY \\ JOHN KARLOF( ${ }^{1}$ )
}

\begin{abstract}
Let $G$ be a finite group and let $H$ be a subgroup of $G$. If $g \in G$, then the set $E_{g}=\left\{h g h^{-1} \mid h \in H\right\}$ is the subclass of $G$ containing $g$ and $\Sigma_{x \in E_{g}} x$ is the subclass sum containing $g$. The algebra over the field of complex numbers generated by these subclass sums is called the subclass algebra (denoted by $S$ ) associated with $G$ and $H$. The irreducible modules of $S$ are demonstrated, and results about Schur algebras are used to develop formulas relating the irreducible characters of $S$ to the irreducible characters of $G$ and $H$.
\end{abstract}

Introduction. Throughout this article $G$ will denote an arbitrary finite group and $H$ a subgroup of $G$. We shall call two elements, $g$ and $g^{\prime}$ of $G, H$-equivalent if there exists an element $h \in H$ such that $g=h g^{\prime} h^{-1}$. Being $H$-equivalent is an equivalence relation on $G$ and the equivalence classes under this relation are called subclasses of $G$. We shall denote the subclass containing the element $g \in G$ by $E_{g}$, and the subclass sum containing $g$ is $B_{g}=\Sigma_{x \in E_{g}} x$. The algebra over the complex numbers $K$ generated by these subclass sums is called the subclass algebra, denoted by $S$, associated with $G$ and $H . S$ is a subalgebra of the group algebra $K G$.

Subclasses were first studied by E. P. Wigner who developed formulas relating the restriction to $H$ of the irreducible characters of $G$ to the number of subclasses. The work done here was started in order to provide a mure algebraic proof to Wigner's results; A paper by F. Roesler about Schur algebras provides an algebraic framework for working with the subclass algebra and proving Wigner's theorems. Roesler's results which are used in this paper are provided for the reader in $\S 1$.

Let $\left\{M_{1} \ldots M_{s}\right\}$ be the irreducible $K G$-modules with $M_{j}$ affording the irreducible character $\chi_{j}$ of $G$ and let $\left\{N_{1} \ldots N_{t}\right\}$ be the irreducible $K H$-modules with $N_{i}$ affording the irreducible character $\Phi_{i}$ of $H$. Suppose $\left\{e_{i}\right\}_{i=1}^{t}$ is a set of orthogonal primitive idempotents of $K H$ such that $N_{i} \simeq K H e_{i}$. Define the nonnegative integers $\left\{c_{i j}\right\}$ by $\left.\chi_{j}\right|_{H}=\Sigma_{i=1}^{t} c_{i j} \Phi_{i}$. Then in $\S 2$ we prove:

Received by the editors April 23, 1973.

AMS(MOS) subject classifications (1970). Primary 20C05; Secondary $20 \mathrm{C1}$.

(1) These results are a part of a Ph.D. thesis written under the direction of Professor

R. L. Roth of the University of Colorado. 
THEOREM (WIGNER). $\Sigma_{i, j}\left(c_{i j}\right)^{2}=$ the number of subclasses.

THEOREM. The irreducible $S$-modules are $\left\{e_{i} M_{j}\right\}$. The dimension of $e_{i} M_{j}$ is $c_{i j}$.

Let $\Psi_{i j}$ be the irreducible character of $S$ afforded by $e_{i} M_{j}$. In $\S 3$, we develop formulas relating the irreducible characters of $S$ to the irreducible characters of $G$ and $H$ and give the relationship between the subclass algebra and the double coset algebra. The following theorem, proved in $\S 3$, may be considered a generalization of a well-known theorem of Clifford:

THEOREM. For $c_{i j} \neq 0$,

$$
\left(\left.\chi_{j}\right|_{H}\right)(h)=\frac{\operatorname{deg} \chi_{j}}{\operatorname{deg} \Phi_{i}|G|} \sum_{k \in G} \Phi_{i}^{*}\left(k h k^{-1}\right)
$$

where

$$
\Phi_{i}^{*}(x)= \begin{cases}\Phi_{i}(x) & \text { if } x \in H, \\ \frac{\operatorname{deg} \Phi_{i}}{c_{i j}\left|E_{x}\right|} \Psi_{i j}\left(B_{x}\right) & \text { if } x \notin H .\end{cases}
$$

In his paper, Wigner observed a number of rather simple but useful properties of subclasses:

(1) Each conjugacy class of $G$ is a union of subclasses.

(2) If a subclass contains an element of $H$, then the subclass is a conjugacy class of $H$.

(3) Each subclass, as a set, commutes with every element of $H$.

(4) For any subclass $E_{g}$, the number of elements in $E_{g}$, denoted $\left|E_{g}\right|$; equals $|H| / C_{H}(g) \mid$, where $C_{H}(g)$ is the subgroup of $H$ consisting of all the elements of $H$ which commute with $g$.

(5) The inverses of the elements of a subclass form a subclass.

(6) The product of two subclass sums is a sum of subclass sums (i.e. $B_{g_{1}} B_{g_{2}}=\Sigma_{g \in G} n_{g} B_{g}$ where the $n_{g}$ are nonnegative integers).

\section{Schur algebras.}

Definition 1.1. Let $A$ be a subalgebra of $K G$. We shall call $A$ a Schur algebra if there exists a partition, $X$, of $G$ such that

(1) The elements $\Sigma_{g \in Y} g(Y \in X)$ form a basis for $A$ over $K$.

(2) If $Y \in X$, then $Y^{*}=\left\{g^{-1} l g \in Y\right\}$ is also in $X$. 
(3) $\{e\} \in X$.

The element of $X$ containing the element $g \in G$ will be denoted $Y_{g}$. We shall also use $\left\{Y_{g}\right\}$ (or just $\{Y\}$ ) to denote the basis elements of $A$. Evidently the subclass algebra is a Schur algebra. In this section $A$ will always be an arbitrary Schur algebra.

$A$ is semisimple [4, Satz 1$]$ so if we let $\left\{\rho_{i}\right\}$ denote the irreducible characters of $A$, we may write $A=\bigoplus_{i} A_{\rho_{i}}$ where the $A_{\rho_{i}}$ are simple two-sided ideals of $A$.

Definition 1.2. $\zeta^{A}: A \rightarrow A$ is defined by $\zeta^{A}(Z)=\Sigma_{Y \in X} c_{Y} Y Z Y^{*}$ where $c_{Y}=v /|Y|$ and $v=\operatorname{LCM}\{|Y| \mid Y \in X\} . C_{0}^{A}=\zeta^{A}\left(Y_{e}\right)$ and $a_{i}=\rho_{i}\left(C_{0}^{A}\right) / \operatorname{deg} \rho_{i}$ for all irreducible characters $\rho_{i}$ of $A$.

TheOREM 1.3 [4, Satz 4]. The central idempotent $e_{i}$ of $A$ such that $A_{\rho_{i}}=$ $A e_{i}$ is

$$
e_{i}=\frac{\operatorname{deg} \rho_{i}}{a_{i}} \sum_{Y} c_{Y} \rho_{i}\left(Y^{*}\right) Y
$$

THEOREM $1.4\left[4\right.$, p. 38]. For any $\rho_{i}$ there exists an irreducible character of $\chi$ of $K G$ such that $\left.\chi\right|_{A}$ contains $\rho_{i}$.

DEFinition 1.5. Let $H^{\prime}$ be a subgroup of $G$ and let $A^{\prime}$ be a Schur algebra of $K H^{\prime}$ with $X^{\prime}$ a partition of $H^{\prime}$ such that $\left\{\Sigma_{g \in Y^{\prime}} g\right\}\left(Y^{\prime} \in X^{\prime}\right)$ is a basis for $A^{\prime}$. Then $A^{\prime}$ is called a Schur subalgebra of $A$ if for each $Y^{\prime}$ there exists a subset $V^{\prime}$ of $X$ such that $Y^{\prime}=\bigcup_{Y \in V^{\prime}} Y$.

Define $w=\operatorname{LCM}\left\{\left|Y^{\prime}\right| \mid Y^{\prime} \in X^{\prime}\right\}$ and $c_{Y^{\prime}}=w /\left|Y^{\prime}\right|$ for $Y^{\prime} \in X^{\prime} .\left\{\gamma_{j}\right\}$ will denote the irreducible characters of $A^{\prime}$. Define $\zeta^{A^{\prime}}$ and $C_{0}^{A^{\prime}}$ in the same manner as we defined $\zeta^{A}$ and $C_{0}^{A}$ and let

$$
b_{j}=\frac{\gamma_{j}\left(C_{0}^{A^{\prime}}\right)}{\operatorname{deg} \gamma_{j}} \text { and } \gamma_{j}^{A}=\frac{w}{v b_{j}} \widetilde{\gamma}_{j} \circ \zeta^{A}
$$

where

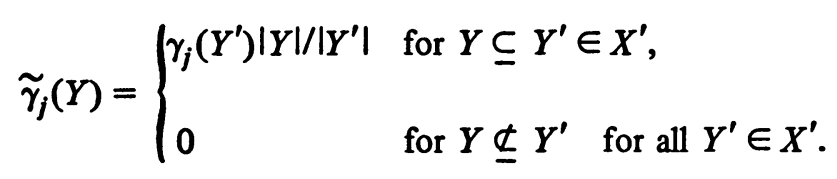

TheOREM 1.6. [4, Satz 6]. $\gamma_{j}^{A}$ is a character of $A$ and if $\rho_{i} I_{A^{\prime}}=\Sigma_{j} d_{i j} \gamma_{j}$, then $\gamma_{j}^{A}=\Sigma_{i} d_{i j} \rho_{i}$. 
Note that the above result is a Frobenius reciprocity theorem for Schur algebras.

THEOREM 1.7 [4, Satz 12]. (a) If $C_{0}^{A} \in A^{\prime}$, then for all $\rho_{i}$ and $\gamma_{j}$ such that $d_{i j} \neq 0$,

$$
\frac{\operatorname{deg} \gamma_{j}^{A}}{\operatorname{deg} \gamma_{j}}=\frac{w}{v} \cdot \frac{a_{i}}{b_{j}} .
$$

(b) All the $\rho_{i}$ in the decomposition of $\gamma_{j}^{A}$ have the same constant $a$.

2. The irreducible $S$-modules.

Proposition 2.1. (a) Let $y$ be an idempotent in $K H$ and let $M$ be a $K G$ module, then $y M$ is an S-module.

(b) If $e$ and $d$ are primitive idempotents in $K H$ such that $K H e \simeq K H d$ (as KH-modules $)$, then $e M_{j} \simeq d M_{j}(j=1 \ldots s)$ as $S$-modules.

Proof. (a) $M$ is a $K G$-module so $M$ is also an $S$-module. Let $x \in S$ and $m \in M$, then since every element of $S$ commutes with every element of $K H$, we have $x(y m)=y(x m) \in y M$ and so $y M$ is an $S$-module.

(b) Consider $P=\operatorname{Hom}_{K H}\left(K H e, M_{i}\right) . P$ can be made into an $S$-module by defining for $a \in K H e, \Psi \in P$, and $x \in S,(x \Psi)(a)=x \Psi(a)$. Now we will show $P$ is $S$-isomorphic to $e M_{i}$.

Define $\Phi: P \longrightarrow e M_{i}$ by $\Phi(f)=f(e)$ for $f \in P . f(e) \in e M_{i}$ since $f(e)=$ $f\left(e^{2}\right)=e f(e)$. Suppose $f, g \in P$ with $\Phi(f)=\Phi(g)$, then for $k e \in K H e(k \in K H)$ we have $f(k e)=k f(e)=k g(e)=g(k e)$ and so $f=g$. So $\Phi$ is one-to-one. $\Phi$ is onto since $\operatorname{dim}(P)=\operatorname{dim}\left(e M_{i}\right)$ [1, Theorem 54.15]. Let $x \in S$, then it is clear that $\Phi(x f)=x \Phi(f)$ and hence $\Phi$ is an $S$-isomorphism.

If $Q=\operatorname{Hom}_{K H}\left(K H d, M_{i}\right)$ where $K H e \simeq K H d$ (as $K H$-modules) then using an argument similar to the one above, $P \simeq Q$ as $S$-modules. So finally we have $e M_{i} \simeq P \simeq Q \simeq d M_{i}$ as $S$-modules. Q.E.D.

In the Introduction, the nonnegative integers $c_{i j}$ were defined by $\left.\chi_{j}\right|_{H}=$ $\Sigma_{i=1}^{t} c_{i j} \Phi_{i}$ where $\left\{\chi_{1} \ldots \chi_{s}\right\}$ are the irreducible characters of $G$ and $\left\{\Phi_{1} \ldots \Phi_{t}\right\}$ are the irreducible characters of $H$.

Corollary 2.2. $\operatorname{dim}\left(e_{i} M_{j}\right)=c_{i j}$.

Proof. By the proof of the preceding proposition, $\operatorname{dim}\left(e_{i} M_{j}\right)=$ $\operatorname{dim}\left(\operatorname{Hom}_{K H}\left(K H e_{i}, M_{j}\right)\right)=c_{i j}$ by Theorem 43.18 in [1]. Q.E.D.

Note that $c_{i j}$ may equal 0 and in this case $e_{i} M_{j}=0$. In the next proposition and elsewhere in this paper, we have the situation where a module $M$ is isomorphic to a direct sum of $a_{1}$ copies of $M_{1}, a_{2}$ copies of $M_{2}, \ldots, a_{n}$ copies of $M_{n}$ for some $n$ and modules $\left\{M_{i}\right\}$. In this case, we shall write $M \approx \sum_{i=1}^{n} a_{i} M_{i}$. 
If $M$ is isomorphic to $m$ copies of a single module $N$, we shall write $M \approx m N$.

$$
\text { Proposition 2.3. }\left.M_{j}\right|_{S} \approx \Sigma_{i=1}^{t}\left(\operatorname{dim} N_{i}\right) e_{i} M_{j} \text {. }
$$

Proof. Let $C_{1} \ldots C_{t}$ be a list of the simple two-sided ideals of $K H$ with each $C_{k}=\bigoplus \Sigma_{l=1}^{\operatorname{dim} N k} K H a_{l, k}$ where $\left\{a_{l, k}\right\}\left(k=1 \ldots t, l=1 \ldots \operatorname{dim} N_{k}\right)$ is a list of orthogonal primitive idempotents. The $S$-modules $\left\{a_{l, k} M_{j}\right\}$ are submodules of $M_{j} l_{s}$. Suppose $a_{l_{1}, k_{1}} m_{1}=a_{l_{2}, k_{2}} m_{2}$ with $l_{1} \neq l_{2}$ or $k_{1} \neq k_{2}$ and $m_{1}$, $m_{2} \in M_{j}$. Then by multiplying by $a_{l_{1}, k_{1}}$ we have $a_{l_{1}, k_{1}} m_{1}=0=a_{l_{2}, k_{2}} m_{2}$. Hence $a_{l_{1}, k_{1}} M_{j} \cap a_{l_{2}, k_{2}} M_{j}=0$. Similarly $a_{l_{1}, k_{1}} M_{j} \cap \Sigma_{(l, k) \neq\left(l_{1}, k_{1}\right)} a_{l, k} M_{j}=0$. Hence $\Sigma_{l, k} a_{l, k} M_{j}$ is a direct sum of $S$-modules.

By Proposition 2.1(b), the primitive central orthogonal idempotents $\left\{e_{i}\right\}_{i=1}^{t}$ may be indexed so that $a_{l, i} M_{j} \simeq e_{i} M_{j}$ (as $S$-modules) for $1 \leqslant l \leqslant \operatorname{dim} N_{i}$. Therefore

$$
\bigoplus \sum_{l, k} a_{l, k} M_{j} \approx \bigoplus \sum_{i=1}^{t}\left(\operatorname{dim} N_{i}\right) e_{i} M_{j} .
$$

By Corollary 2.2, $\operatorname{dim} e_{i} M_{j}=c_{i j}$ and so we have that $\Sigma_{i=1}^{t}\left(\operatorname{dim} N_{i}\right) e_{i} M_{j}$ is a submodule of $\left.M_{j}\right|_{S}$ of dimension $\Sigma_{i=1}^{t}\left(\operatorname{dim} N_{i}\right) c_{i j}$. But since $\left.M_{j}\right|_{K H} \approx \Sigma_{i=1}^{t} c_{i j} N_{i}$, it follows that $\operatorname{dim} M_{j}=\Sigma_{i=1}^{t}\left(\operatorname{dim} N_{i}\right) c_{i j}$. Consequently we have $\left.M_{j}\right|_{S} \approx$ $\Sigma_{i=1}^{t}\left(\operatorname{dim} N_{i}\right) e_{i} M_{j}$. Q.E.D.

We are now ready to prove the first of Wigner's formulas in [7]. Let $z=$ the number of subclasses of $G$.

THEOREM 2.4. $\Sigma_{i, j}\left(c_{i j}\right)^{2}=z$.

In order to prove Theorem 2.4 , the following lemma will be used:

LEMMA. $z=|H|^{-1} \Sigma_{h \in H}\left|C_{G}(h)\right|$.

Proof of the Lemma. Since $\Sigma_{g \in G}\left|C_{H}(g)\right|=\Sigma_{h \in H}\left|C_{G}(h)\right|$ we have

$$
\begin{aligned}
|H|^{-1} \sum_{h \in H}\left|C_{G}(h)\right| & =\sum_{g \in G} \frac{\left|C_{H}(g)\right|}{|H|} \\
& =\sum_{g \in E_{1}} \frac{1}{\left|E_{1}\right|}+\ldots+\sum_{g \in E_{z}} \frac{1}{\left|E_{z}\right|}=z,
\end{aligned}
$$

where $\left\{E_{1} \ldots E_{z}\right\}$ are the subclasses of $G$. Q.E.D.

ProOF OF THEOREM 2.4. Let $U$ be the $K$-vector space of class functions of $G$ and $U^{\prime}$ be the $K$-vector space of class functions of $H$. Following Winter [8], define $T_{1}: U \rightarrow U^{\prime}$ by $T_{1}(\theta)=\left.\theta\right|_{H}$ and define $T_{2}: U^{\prime} \rightarrow U$ by $T_{2}(\Psi)=\Psi^{G}$. Then $T_{1}$ and $T_{2}$ are linear transformations. Let $A_{1}$ be the matrix for $T_{1}$ and $A_{2}$ be the matrix for $T_{2}$ taking for bases of $U$ and $U^{\prime}$ the irreducible characters of 
$G$ and $H$ respectively. Then $A_{1}=\left(c_{i j}\right)(i=1 \ldots t, j=1 \ldots s)$ and $A_{2}=$ the transpose of $A_{1}$. Let $T: U \rightarrow U$ be defined by $T(\theta)=\left(\left.\theta\right|_{H}\right)^{G}=T_{2} \circ T_{1}(\theta)$. So the matrix for $T$ with respect to the basis of irreducible characters of $G$ for $U$ is

$$
A=A_{2} A_{1}=\left(\begin{array}{cccc}
\Sigma_{i=1}^{t} c_{i 1}^{2} & & \cdots & \\
\vdots & \Sigma_{i=1}^{t} c_{i 2}^{2} & & \vdots \\
\cdots & & \Sigma_{i=1}^{t} c_{i s}^{2}
\end{array}\right)
$$

Another basis for $U$ is $\left\{f_{1} \ldots f_{s}\right\}$ defined by

$$
f_{i}(g)= \begin{cases}0 & \text { if } g \notin K_{i}, \\ 1 & \text { if } g \in K_{i},\end{cases}
$$

where $\left\{K_{1} \ldots K_{s}\right\}$ is the set of conjugacy classes of $G$. Choose $k$ such that $K_{i} \cap H \neq \varnothing \quad(1 \leqslant i \leqslant k)$ and $K_{i} \cap H=\varnothing$ for $i>k$. Let

$$
r_{i}=\left|C_{G}\left(g_{i}\right)\right|\left|K_{i} \cap H\right| / H \mid
$$

where $g_{i} \in K_{i}$. It can be easily shown that the matrix for $T$ with respect to the new basis $\left\{f_{1} \ldots f_{s}\right\}$ of $U$ is

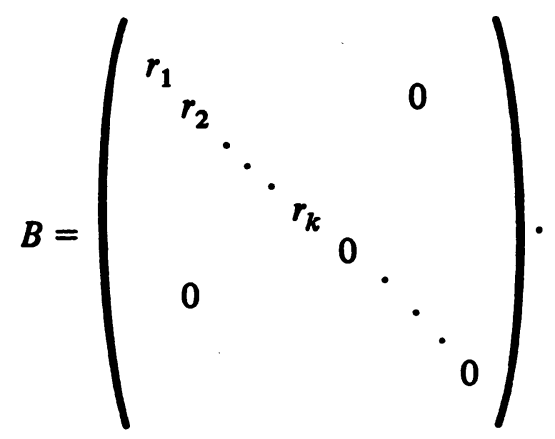

But

$$
\begin{aligned}
\sum_{i=1}^{k} r_{i} & =|H|^{-1} \sum_{i=1}^{k}\left|C_{G}\left(g_{i}\right)\right| \cdot\left|K_{i} \cap H\right| \\
& =|H|^{-1} \sum_{j=1}^{t}\left|C_{G}\left(h_{j}\right)\right| \cdot\left|C_{j}\right| \quad \text { where the conjugacy classes of } H \\
& =|H|^{-1} \sum_{h \in H}\left|C_{G}(h)\right| \\
& =z \text { are }\left\{C_{1} \ldots C_{t}\right\} \text { with } h_{j} \in C_{j} . \\
& \text { by the Lemma. }
\end{aligned}
$$


However, since $T$ is a linear transformation we have $\operatorname{trace}(A)=\operatorname{trace}(B)$ and therefore $\Sigma_{i, j}\left(c_{i j}\right)^{2}=z$. Q.E.D.

THEOREM 2.5. The irreducible $S$-modules are $\left\{e_{i} M_{j}\right\}(i=1 \ldots t, j=1 \ldots s)$ and if $e_{i} M_{j} \neq 0$ then $e_{i} M_{j} \mp e_{i^{\prime}} M_{j^{\prime}}$ for $(i, j) \neq\left(i^{\prime}, j^{\prime}\right)$.

Proof. Let $\left\{V_{1} \ldots V_{l}\right\}$ be the irreducible $S$-modules. By Theorem 1.4 and Proposition 2.3, for each $k(1 \leqslant k \leqslant l)$ we may choose an $e_{i_{k}} M_{j_{k}}$ such that $V_{k}$ is a submodule of $e_{i_{k}} M_{j_{k}}$. By Corollary $2.2, c_{i_{k} j_{k}}=\operatorname{dim} e_{i_{k}} M_{j_{k}}$. If for some $k, e_{i_{k}} M_{j_{k}}=n V_{k}$ then $c_{i_{k} j_{k}} \geqslant \operatorname{dim} V_{k}$ and so $\left(c_{i_{k} j_{k}}\right)^{2} \geqslant\left(\operatorname{dim} V_{k}\right)^{2}$. And if for some $k, e_{i_{k}} M_{j_{k}}=\Sigma_{p} n_{p} V_{p}$ then $c_{i_{k} j_{k}} \geqslant \Sigma_{p} \operatorname{dim} V_{p}$ and so $\left(c_{i_{k} j_{k}}\right)^{2} \geqslant \Sigma_{p}\left(\operatorname{dim} V_{p}\right)^{2}$. Suppose there exists a $k^{\prime}$ such that $c_{i_{k^{\prime}}, j_{k^{\prime}}}>\operatorname{dim} V_{k^{\prime}}$. By Theorem 2.4, the number of subclasses $=\Sigma_{i, j}\left(c_{i j}\right)^{2} \geqslant \Sigma^{\prime}\left(c_{i_{k} j_{k}}\right)^{2}>\Sigma_{k=1}^{l}\left(\operatorname{dim} V_{k}\right)^{2}=$ the number of subclasses which is impossible ( $\Sigma^{\prime}$ is summed only over those $i_{k}, j_{k}$ 's chosen above without repeating any). Hence $c_{i_{k} j_{k}}=\operatorname{dim} V_{k}(1 \leqslant k \leqslant l)$ and $\left\{e_{i} M_{j}\right\}$ are exactly the irreducible $S$-modules. Q.E.D.

COROLlaRY 2.6. $\left.\chi_{j}\right|_{S}$ is irreducible if and only if $\left.\chi_{j}\right|_{H}=n \Phi$ for a one-dimensional character $\Phi$ of $H$.

PRoof. Proposition 2.3 and Theorem 2.5. Q.E.D.

The next corollary was proved by Wigner as a main result of [7]. Let

$$
c_{i j}^{\epsilon}= \begin{cases}1 & \text { if } c_{i j}>0, \\ 0 & \text { if } c_{i j}=0 .\end{cases}
$$

Define a commutator of $S$ to be an element of the form $x y-y x$ for $x, y \in S$.

COROLlaRY 2.7. $\Sigma_{i, j} c_{i j}^{\epsilon}=$ the number of subclasses minus the number of linearly independent commutators of these.

Proof. By Theorem 2.5, $\Sigma_{i, j} c_{i j}^{\epsilon}=$ the number of distinct irreducible characters of $S$. But as is well known, the number of distinct irreducible characters = $\operatorname{dim}(Z(S))=\operatorname{dim}(S)-\operatorname{dim}\left(S^{\prime}\right)$ where $S^{\prime}$ is the subspace of $S$ generated by commutators of $S$. Q.E.D.

3. The irreducible characters of $S$. In this section, we will use the information about Schur algebras discussed in $\S 1$. Recall that $S$ and $K G$ are Schur algebras of $K G$ and $Z(K H)$ is a Schur algebra of $K H$. We may also view $S$ as a Schur subalgebra of the Schur algebra $K G$, since each subclass is a union of basis elements of $K G$. Since the conjugacy classes of $H$ are subclasses, $Z(K H)$ is a Schur subalgebra of $S$. 
Theorem 2.5 identified the irreducible $S$-modules as $\left\{e_{i} M_{j}\right\}$ where $\left\{M_{1} \ldots M_{s}\right\}$ are the irreducible $K G$-modules and $\left\{e_{1} \ldots e_{t}\right\}$ is a set of orthogonal primitive idempotents of $K H$. It will be understood that $e_{i} M_{j}$ appears in the list of irreducible $S$-modules if and only if $c_{i j} \neq 0$. Let $\left\{\Psi_{i j}\right\}$ be the irreducible characters of $S$ with $\Psi_{i j}$ afforded by $e_{i} M_{j}$. Proposition 2.3 implies that $\chi_{j} l_{S}=\Sigma_{i}\left(\operatorname{deg} \Phi_{i}\right) \Psi_{i j}$. So $\Psi_{i j 0}$ is a component of $\chi_{j_{0}} l_{S}$ and is not a component of $\left.\chi_{k}\right|_{s}$ for $k \neq j_{0}$.

Let $Z(K H)$ be the center of $K H$ and $\left\{w_{i}\right\}_{i=1}^{t}$ the irreducible characters of $Z(K H)$ defined by $w_{i}\left(C_{h}\right)=\left|C_{h}\right| \Phi_{i}(h) / \operatorname{deg} \Phi_{i}(i=1 \ldots t)$ where $C_{h}$ is the conjugacy class of $H$ containing the element $h$ of $H\left[2\right.$, p. 28]. The characters $\left\{\Phi_{i}\right\}$ are indexed so that $\Phi_{i}$ is the character afforded by the irreducible $K H$-module $\mathrm{KHe}_{\boldsymbol{i}}$.

THEOREM 3.1. $\left.\Psi_{i j}\right|_{Z(K H)}=c_{i j} w_{i}$.

Proof. Let

$$
f_{i}=\frac{\operatorname{deg} \Phi_{i}}{|H|} \sum_{C_{h}} \Phi_{i}\left(h^{-1}\right) C_{h}
$$

[3, p. 483] be the orthogonal central idempotents of $Z(K H)$. So $Z(K H) \simeq$ $\bigoplus \Sigma_{i=1}^{t} Z(K H) f_{i}$ where $\left\{Z(K H) f_{i}\right\}$ are the simple two-sided ideals of $Z(K H)$ and also the irreducible $Z(K H)$-modules since they are of dimension one.

Let $e_{i} M_{j} I_{Z(K H)} \approx \Sigma_{k=1}^{t} n_{k}^{i j}\left(Z(K H) f_{k}\right)$ where the $n_{k}^{i j}$ are nonnegative integers. Since $f_{i} e_{i}=e_{i}$, it follows that $f_{i} e_{i} M_{j} \simeq e_{i} M_{j}(i=1 \ldots t)$. Now, multiplication of both sides of the first equation in this paragraph by $f_{i}$ yields $\left.e_{i} M_{j}\right|_{Z(K H)} \approx n_{i}^{i j}\left(Z(K H) f_{i}\right)$. This implies

$$
n_{k}^{i j}= \begin{cases}0 & \text { if } k \neq i, \\ c_{i j} & \text { if } k=i,\end{cases}
$$

since $\operatorname{dim}\left(e_{i} M_{j}\right)=c_{i j}$ and $\operatorname{dim}\left(Z(K H) f_{k}\right)=1$. Hence $\left.\Psi_{i j}\right|_{Z(K H)}=c_{i j} w_{i}$. Q.E.D.

$Z(K H)$ is a Schur subalgebra of $S$, so we may induce the irreducible characters $w_{i}$ of $Z(K H)$ to characters $w_{i}^{S}$ of $S$.

COROLLARY 3.2. $w_{i}^{S}$ is an irreducible character of $S$ if and only if there exists $a j$ such that $c_{i j}=1$ and $c_{i k}=0$ for $k \neq j$.

Proof. By Theorem 3.1, $\Psi_{i j} I_{Z(K H)}=c_{i j} w_{i}$ for all $i$ and $j$. So by Theorem $1.6, w_{i}^{S}=\Sigma_{j} c_{i j} \Psi_{i j}$ and $w_{i}^{S}$ is irreducible if and only if one $c_{i j}$ in the sum is nonzero and this $c_{i j}$ has to be 1. Q.E.D. 
In $\S 1$, integer constants $v, c_{y}$, and $a_{i}$ were defined associated with a Schur algebra. Also the constant $C_{0}^{A}$ of a Schur algebra $A$ was defined. In Proposition 3.3, we give the values for these constants for the Schur algebra $S$. Note

$$
\begin{array}{rlrl}
v & =\operatorname{LCM}\left\{\left|E_{g}\right| \mid g \in G\right\} \quad \text { and } \quad c_{B_{g}}=\frac{v}{\left|E_{g}\right|}, \\
C_{0}^{S} & =\sum_{B_{g}} c_{B_{g}} B_{g} B_{g}^{*} & \text { and } \quad a_{i j} & =\frac{\Psi_{i j}\left(C_{0}^{S}\right)}{\operatorname{deg} \Psi_{i j}} .
\end{array}
$$

Proposition 3.3.

$$
\begin{gathered}
C_{0}^{S}=\frac{v}{|H|} \sum_{h \in H ; g \in G} g h g^{-1} h^{-1}, \\
a_{i j}=\frac{v|G| c_{i j}}{\operatorname{deg} \Phi_{i} \operatorname{deg} \chi_{j}}
\end{gathered}
$$

Proof. (a) $C_{0}^{S}$ was defined to be $\Sigma_{B_{g}}\left(v /\left|E_{g}\right|\right) B_{g} B_{g}^{*}$. Choose a $B_{g_{1}}$ and let $B_{g_{1}}=g_{1}+\ldots+g_{n}$, then $B_{g_{1}}^{*}=g_{1}^{-1}+\ldots+g_{n}^{-1}$. For $g, g^{\prime} \in B_{g_{1}}$ there exists an $h \in H$ such that $h C_{H}(g) h^{-1}=C_{H}\left(g^{\prime}\right)$. For each $i$ with $1 \leqslant i \leqslant n$, there exists a subset $\left\{h_{i 1}, \ldots, h_{i n}\right\}$ of $H$ such that $H=h_{i 1} C_{H}\left(g_{i}\right) \cup \ldots \cup h_{i n} C_{H}\left(g_{i}\right)$ and we may write

$$
B_{g_{1}}^{*}=g_{1}^{-1}+h_{12} g_{1}^{-1} h_{12}^{-1}+\ldots+h_{1 n} g_{1}^{-1} h_{1 n}^{-1}
$$

or

$$
B_{g_{1}}^{*}=g_{2}^{-1}+h_{22} g_{2}^{-1} h_{22}^{-1}+\ldots+h_{2 n} g_{2}^{-1} h_{2 n}^{-1}
$$

or

$$
B_{g_{1}}^{*}=g_{n}^{-1}+h_{n 2} g_{n}^{-1} h_{n 2}^{-1}+\ldots+h_{n n} g_{n}^{-1} h_{n n}^{-1} \text {. }
$$

So

$$
\begin{aligned}
B_{g_{1}} B_{g_{1}}^{*}= & \left(1+g_{1} h_{12} g_{1}^{-1} h_{12}^{-1}+\ldots+g_{1} h_{1 n} g_{1}^{-1} h_{1 n}^{-1}\right) \\
& +\left(1+g_{2} h_{22} g_{2}^{-1} h_{22}^{-1}+\ldots+g_{2} h_{2 n} g_{2}^{-1} h_{2 n}^{-1}\right) \\
& +\ldots+\left(1+g_{n} h_{n 2} g_{n}^{-1} h_{n 2}^{-1}+\ldots+g_{n} h_{n n} g_{n}^{-1} h_{n n}^{-1}\right) \\
= & \frac{1}{\left|C_{H}\left(g_{1}\right)\right|}\left[\sum_{n \in H} g_{1} h g_{1}^{-1} h^{-1}+\ldots+\sum_{h \in H} g_{n} h g_{n}^{-1} h^{-1}\right]
\end{aligned}
$$


Hence $\left|E_{g}\right|^{-1} B_{g} B_{g}^{*}=|H|^{-1} \Sigma_{h \in H ; k \in E_{g}} k h k^{-1} h^{-1}$ for each $g \in G$ and so $C_{0}^{S}=$ $v|H|^{-1} \Sigma_{h \in H: g \in G} g h g^{-1} h^{-1}$. Q.E.D.

(b) By the discussion in the beginning of this section, the only irreducible character of $G$ with the property that $\Psi_{i j}$ appears in its restriction to $S$ is $\chi_{j}$ and $\Psi_{i j}$ appears with multiplicity $\operatorname{deg} \Phi_{i}$. So $\Psi_{i j}^{K G}=\left(\operatorname{deg} \Phi_{i}\right) \chi_{j}$ by Theorem 1.6. This implies $\operatorname{deg} \Psi_{i j}^{K G}=\left(\operatorname{deg} \Phi_{i}\right)\left(\operatorname{deg} \chi_{j}\right)$. Letting $K G=$ the Schur algebra $A$ in the definitions of $\S 1$, we have $C_{0}^{K G}=|G|$ and the constants $b_{j}=\chi_{j}\left(C_{0}^{K G}\right) / \mathrm{deg} \chi_{j}$ $=|G|$. By Theorem 1.7(a),

$$
\frac{\operatorname{deg} \Psi_{i j}^{K G}}{\operatorname{deg} \Psi_{i j}}=\frac{v b_{j}}{a_{i j}} \text { or } \frac{\operatorname{deg} \Phi_{j} \operatorname{deg} \chi_{j}}{\operatorname{deg} \Psi_{i j}}=\frac{v|G|}{a_{i j}} \text {. Q.E.D. }
$$

Proposition 3.4.

$$
\sum^{\prime}\left(\operatorname{deg} \chi_{j}\right) \Psi_{i j}\left(B_{g}\right)=0 \text { for } B_{g} \cap H=\varnothing
$$

and

$$
\sum^{\prime}\left(\operatorname{deg} \chi_{j}\right) \Psi_{i j}\left(B_{g}\right)=|G||H|^{-1} \Phi_{i}\left(B_{g}\right) \text { for } B_{g} \cap H \neq \varnothing .
$$

The sum $\Sigma^{\prime}$ means we sum over all $j$ 's such that $e_{i} M_{j} \neq 0$.

Proof. As in the proof of Theorem 3.1, let

$$
f_{i}=\frac{\operatorname{deg} \Phi_{i}}{|H|} \sum_{B_{h} \subseteq H} \Phi_{i}\left(h^{-1}\right) B_{h} \quad \text { for } i=1 \ldots t
$$

be the central primitive idempotents of $K H$. (We may sum over the subclasses contained in $H$ because they are exactly the conjugacy classes of $H$.) Since each element of $S$ commutes with all the elements of $K H$, each $f_{i}$ is also a central idempotent of $S$. Let $\left\{b_{l}\right\}$ be the primitive central idempotents of $S$ indexed such that there exist disjoint sets $\left\{b_{l}\right\}_{l=1}^{n_{1}},\left\{b_{l}\right\}_{l=n_{1}+1}^{n_{2}}, \ldots,\left\{b_{l}\right\}_{l=n_{t-1} n_{t}}^{n_{i}}$ such that $f_{i}=$ $\Sigma_{l=n_{i-1}+1}^{n_{i}} b_{l}$.

The simple two-sided ideals of $S$ are $\left\{S b_{l}\right\}$ and each $S b_{l}$ is the direct sum of isomorphic $S$ modules. We will show that if $n_{i_{0}-1}<l \leqslant n_{i_{0}}$ for some $i_{0}$ and if $S b_{l} \approx\left(\operatorname{dim} e_{i} M_{j}\right) e_{i} M_{j}$ for some $i$ and $j$, then necessarily $K H f_{i_{0}} \approx\left(\operatorname{dim} K H e_{i}\right) K H e_{i}$. So suppose $e_{i} M_{j}$ is a direct summand of $S b_{l}$ for $n_{i_{0}-1}<l \leqslant n_{i_{0}}$ and $K H e_{i}$ is not a direct summand of $K H f_{i_{0}}$. Then $0=S\left(f_{i_{0}} b_{l}\right)$ since in this case $f_{i_{0}}$ annihilates $e_{i} M_{j}$. But this implies $f_{i_{0}} b_{l}=0$ which is impossible.

Hence we may renumber the $b_{l}$ 's with two subscripts such that $S b_{i j} \approx$ $\left(\operatorname{dim} e_{i} M_{j}\right) e_{i} M_{j}$ and $b_{i j}$ is a summand of $f_{i}$. Now by Theorem 1.3,

$$
b_{i j}=\frac{\operatorname{deg} \Psi_{i j}}{a_{i j}} \sum_{B_{g}} c_{B_{g}} \Psi_{i j}\left(B_{g}^{*}\right) B_{g}
$$


and hence

$$
\begin{aligned}
\frac{\operatorname{deg} \Phi_{i}}{|H|} \sum_{B_{h} \subseteq H} \Phi_{i}\left(h^{-1}\right) B_{h} & =\sum_{j} \frac{\operatorname{deg} \Psi_{i j}}{a_{i j}} \sum_{B_{g}} c_{B_{g}} \Psi_{i j}\left(B_{g}^{*}\right) B_{g} \\
& =\sum_{B_{g}} \frac{1}{\left|E_{g}\right|}\left[\sum_{j} \frac{\operatorname{deg} \Phi_{i} \operatorname{deg} \chi_{j}}{|G|} \Psi_{i j}\left(B_{g}^{*}\right)\right] B_{g}
\end{aligned}
$$

by Proposition 3.3(b). So by comparing coefficients, we have for $B_{g} \cap H=\varnothing$,

$$
0=\frac{1}{\left|E_{g}\right|} \frac{\operatorname{deg} \Phi_{i}}{|G|} \sum_{j}\left(\operatorname{deg} \chi_{j}\right) \Psi_{i j}\left(B_{g}\right)
$$

therefore $\Sigma_{j}\left(\operatorname{deg} \chi_{j}\right) \Psi_{i j}\left(B_{g}\right)=0$ and, for $B_{g} \cap H \neq \varnothing$,

$$
\sum_{j}\left(\operatorname{deg} \chi_{j}\right) \Psi_{i j}\left(B_{g}\right)=|G||H|^{-1} \Phi_{i}\left(B_{g}\right) \text {. Q.E.D. }
$$

THEOREM 3.5. For any $c_{i j}$ that is not equal to zero we have for $g \in G$,

$$
\chi_{j}(g)=\frac{\operatorname{deg} \chi_{j}}{c_{i j}\left|K_{g}\right|} \Psi_{i j}\left(K_{g}\right)
$$

where $K_{g}$ is the conjugacy class of $G$ containing $g$.

Proof. By the discussion in the introduction to this section, $\Psi_{i j}$ is a compotent of $\left.\chi_{k}\right|_{s}$ if and only if $k=j$ and $c_{i j} \neq 0$ and if this is the case $\Psi_{i j}$ occurs as a component of $\left.\chi_{j}\right|_{s}$ with multiplicity $\operatorname{deg} \Phi_{i}$. Therefore, by Theorem 1.6,

$$
\left(\operatorname{deg} \Phi_{i}\right) x_{j}=\Psi_{i j}^{K G}=\left(v / a_{i j}\right) \widetilde{\Psi}_{i j} \circ \zeta^{K G}
$$

where

$$
\widetilde{\Psi}_{i j}(g)=\left|E_{g}\right|^{-1} \Psi_{i j}\left(B_{g}\right) \text { and } \zeta^{K G}(g)=\sum_{k \in G} k g k^{-1} .
$$

So for $g \in G$ we have by Proposition 3.3(b),

$$
\left(\operatorname{deg} \Phi_{i}\right) \chi_{j}(g)=\frac{v \operatorname{deg} \Phi_{i} \operatorname{deg} \chi_{j}}{v|G| c_{i j}} \widetilde{\Psi}_{i j} \circ \zeta^{K G}(g)
$$

and hénce

$$
\begin{aligned}
\chi_{j}(g) & =\frac{\operatorname{deg} \chi_{j}}{|G| c_{i j}} \sum_{k \in G} \frac{1}{\left|E_{k g k^{-1}}\right|} \Psi_{i j}\left(B_{k g k^{-1}}\right) \\
& =\frac{\operatorname{deg} \chi_{j}}{|G| c_{i j}}\left|C_{G}(g)\right| \sum_{k \in K_{g}} \frac{1}{\left|E_{k}\right|} \Psi_{i j}\left(B_{k}\right)=\frac{\operatorname{deg} \chi_{j}}{c_{i j}\left|K_{g}\right|} \Psi_{i j}\left(K_{g}\right) \text {. Q.E.D. }
\end{aligned}
$$


COROLlary 3.6. For $h \in H$, and $c_{i j} \neq 0$,

$$
\left.\chi_{j}\right|_{H}(h)=\frac{\operatorname{deg} \chi_{j}}{\operatorname{deg} \Phi_{i}|G|} \sum_{k \in G} \Phi_{i}^{*}\left(k h k^{-1}\right)
$$

where

$$
\Phi_{i}^{*}(x)= \begin{cases}\Phi_{i}(x) & \text { if } x \in H, \\ \frac{\operatorname{deg} \Phi_{i}}{c_{i j}\left|E_{x}\right|} \Psi_{i j}\left(B_{x}\right) & \text { if } x \notin H .\end{cases}
$$

Proof. By the proof of Theorem 3.5 and Theorem 3.1,

$$
\begin{aligned}
\chi_{j}(h)=\frac{\operatorname{deg} \chi_{j}}{|G| c_{i j}}\left[\sum_{k \in G ; k h k^{-1} \in H} c_{i j} \frac{\Phi_{i}\left(k h k^{-1}\right)}{\operatorname{deg} \Phi_{i}}\right. & \\
& \left.+\sum_{k \in G ; k h k^{-1} \notin H} \frac{1}{\left|E_{k h k^{-1}}\right|} \Psi_{i j}\left(B_{k h k^{-1}}\right)\right] \text {. Q.E.D. }
\end{aligned}
$$

Note that if $H \triangleleft G$, Corollary 3.6 is Theorem $17.3(\mathrm{~g})$ in [3].

THEOREM (CLIFFORD). If $\chi$ is an irreducible character of $G$ and $N$ is a normal subgroup of $G$, then all the irreducible characters of $N$ which are components of $\left.\chi\right|_{N}$ are conjugate. That is, $\left.\chi\right|_{N}=e \sum_{i=1}^{n} \Phi^{g_{i}}$ where $\Phi^{x}(g)=\Phi\left(x g x^{-1}\right)$, $\left\{g_{1} \ldots g_{n}\right\}$ are coset representatives of the subgroup $\left\{k \in G \mid \Phi^{k}=\Phi\right\}$ of $G$ and $e=(\operatorname{deg} \chi) / n \operatorname{deg} \Phi$.

So we may think of Corollary 3.6 as a generalization of this theorem of Clifford.

O. Tamaschke [5], [6] has done considerable work on Schur algebras, but unlike Roesler, he does not require $\{e\}$ to be in the partition of $G$ which forms the basis elements for the algebra. An algebra Tamaschke has given special attention to is the double coset algebra which is the subalgebra of $K G$ generated by all double cosets, $\mathrm{HgH}$, with $g \in G$. By modifying a proof of Tamaschke, [5, p. 19] it can be shown that the double coset algebra is a two-sided ideal of the subclass algebra.

\section{REFERENCES}

1. C. Curtis and I. Reiner, Representation theory of finite groups and associative algebras, Pure and Appl. Math., vol. 11, Interscience, New York, 1962. MR 26 \#2519.

2. W. Feit, Characters of finite groups, Benjamin, New York and Amsterdam, 1967. MR 36 \#2715.

3. B. Huppert, Endliche Gruppen. I, Die Grundlehren der math. Wissenschaften, Band 
134, Springer-Verlag, Berlin and New York, 1967. MR 37 \#302.

4. F. Roesler, Darstellungstheorie von Schur-Algebren, Math. Z. 125 (1972), 32-58. MR 46 \#1892.

5. O. Tamaschke, On the theory of Schur-rings, Ann. Mat. Pura Appl. (4) 81 (1969), 1-43. MR 39 \#5721.

6. - S-rings and the irreducible representations of finite groups, J. Algebra 1 (1964), 215-232. MR 29 \#4813.

7. E. Wigner, Restriction of irreducible representations of groups to a subgroup, Proc. Roy. Soc. London Ser. A 322 (1971), 181-189.

8. D. Winter, On the restrict-induce map of group characters, Proc. Amer. Math. Soc. 19 (1968), 246-248. MR 37 \#326.

DEPARTMENT OF MATHEMATICS, UNIVERSITY OF NEBRASKA AT OMAHA, OMAHA, NEBRASKA 68101 